\title{
NUTRIENTES MINERALES EN ALIMENTOS INDUSTRIALIZADOS
}

\author{
HERNÁNDEZ F. ELOISA, QUISPE CLARA*, ALENCASTRE M. ANA**
}

Facultad de Farmacia y Bjoquímica de la Universidad Nacional Mayor de San Marccos Cátedra de Bromatología y Nutricion Aplicada

\section{RESUMEN}

Hasta el momento los alimentos industrializados que se consumen en nuestro medio aún no han sido estudiados por su contenido de nutrientes minerales. Se presenta los contenidos de fósforo, calcio, hierro, sodio, potasio, cobre y magnesio en 33 alimentos de procedencia nacional e importados. El $70 \%$ de estos alimentos son preferentemente consumidos por niños.

Se ha empleado métodos oficiales de A.O.A.C.: para fósforo el método colorimétrico, los otros minerales mediante Absorción Atómica, Espectrofotómetro de P.E. Mod. 305. Las gráficas de respuestas a diferentes concentraciones de minerales presentan valores de $r=0,97, P>0,001$.

En productos dietéticos deshidratados $(n=7)$, el hierro se encontró entre 2,9 a $11 \mathrm{mg} \%$; calcio 378 a $550 \mathrm{mg} \%$. Los derivados de cereales $(n=8)$ : sodio de 14 a $948 \mathrm{mg} \%$; potasio, 83 a 345 $\mathrm{mg} \%$; magnesio 4,9 a $453 \mathrm{mg} \%$ y fósforo 43 a $243 \mathrm{mg} \%$. En néctares $(n=11)$; potasio de 16 a $99 \mathrm{mg} \%$, calcio de 10 a $30 \mathrm{mg} \%$ y 1,7 a $4,5 \mathrm{mg} \%$ de magnesio

Los jugos $(n=7)$ contenían sodio de 6 a $88 \mathrm{mg} \%$; potasio de 4 a $72 \mathrm{mg} \%$ y 3 a $8 \mathrm{mg} \%$ de magnesio.

La frecuencia de consumo de este tipo de alimentos determinarian que sean importantes fuentes de macro y micronutrientes minerales y que por la naturaleza y composición integral de aquellos, dichos minerales resultarian altamente biodisponibles.

Los datos indican la necesidad de investigar el contenido de nutrientes minerales en alimentos para conocer los verdaderos aportes nutricionales a las dietas de las poblaciones.

\section{INTRODUCCIÓN}

Recientemente ha sido reconocido que no existen datos, que éstos son insuficientes, con respecto al contenido de nutrientes minerales, en alimentos que son de consumo habitual entre los pobladores. Este es el caso de los alimentos procesados, cuyo conocimiento sobre su composición en minerales es imprescindible para poder evaliar resultados de las ingestas dietarias y determinar la idoneidad de su consumo por parte de la población.

Con la finalidad de aportar información, que permita un mejor conocimiento sobre el estado de nutrición en nutrientes minerales, se investigó en cuatro grupos de alimentos procesados el contenido de fósforo, calcio, cobre, hierro, sodio, potasio y magnesio.

Este trabajo se realizó con recursos propios de los investigadores y apoyo Técnico y Logístico del Instituto Nacional de Salud y la Dirección de Protección de Alimentos de la Sub-Región de Salud - Callao.

- Investigador en el I.N.S.

.- Investigador, Director Ejecutivo de Salud Ambiental, DISURS 1 Callao

Los autores agradecen la oportunidad de publicación del trabajo al Consejo Stuperior de Investigación, Facultad de Famnacia y Bioquimica de la U.N.M.S.M.

A la Mg. Jesús Rumiche, Cátedra de Salud Pública de la Facultad de Farmacia y Bioquimica por el apoyo en la sistematización de! trabajo. 


\section{MATERIALES Y MÉTODOS}

Muestras: 7 productos dietéticos, 8 derivados de cereales, 11 néctares y 7 jugos de frutas. Fueron proporcionadas - por duplicado- por un laboratorio de control de calidad sanitaria.

Del total de 33 alimentos, 13 eran de procedencia extranjera, los restantes eran de procedencia nacional. Todos habían cumplido los estándares físicos, químicos y microbiológicos de las normas sanitarias. Cada una de las muestras fue procesada por duplicado, para determinar previamente el contenido de agua, por gravimetría. y posteriormente el contenido total de minerales, por incineracion (1).

Para la determinación de fósforo se empleó el método espectrofotométrico descrito por la A.O.A.C. (1).

El calcio, hierro, cobre, sodio, potasio y magnesio fueron determinados por espectrofotometría de Absorción Atómica empleando el espectrofotómetro de Perkin Elmer Mod. 305.

La investigación se llevó a cabo en el año 1992. Hasta la fecha en que es remitodo el artículo para su publicación, los 33 alimentos estudiados, aún se comercializan en nuestro mercado.

\section{RESULTADOS}

Se presentan en cuatro tablas:

Tabla Na 1.- Productos dietéticos en polvo $(\mathbf{n}=7)$

Estos productos destacan por su elevado contenido de calcio (358 a $546 \mathrm{mg} \%$ ), potasio (414 a $600 \mathrm{mg} \%$ ) y fósforo (170 a $350 \mathrm{mg} \%$ ).

Tabla N ${ }^{a}$ 2.- Derivados de cereales $(n=8)$

Entre las muestras de fideos, el enriquecido con huevo presentó los mayores niveles para: fósforo (243 $\mathrm{mg} \%$ ), calcio (178\% $\mathrm{mg} \%$ ), sodio (948 $\mathrm{mg} \%$ ) y magnesio (453 $\mathrm{mg} \%$ ).

En la sémola, el contenido de sodio fue de $498 \mathrm{mg} \%$ y los valores de cobre $(0,067$ $\mathrm{mg} \%$ ) fueron los más bajos en relación a los demás nutrientes.

En la harina de maíz, el contenido de calcio fue de $118 \mathrm{mg} \%$ y el de cobre 0.3 $\mathrm{mg} \%$.

Tabla Na 3.- Néctares $(\mathrm{n}=11)$

Con excepción de los valores de sodio ( 8 a $152 \mathrm{mg} \%$ ), los valores de $\mathrm{Ca}, \mathrm{Fe}, \mathrm{Cu}$, k y $\mathrm{Mg}$ eran muy similares entre sí. Los contenidos de $\mathrm{Cu}(0,03$ a $0,11 \mathrm{mg} \%)$ y de $\mathrm{Fe}$ $(0,19$ a $0,54 \mathrm{mg} \%)$ fueron los valores más equeños en comparación con los restantes minerales.

Este tipo de productos, destaca por el contenido de sodio ( 8 a $152 \mathrm{mg} \%$ ) y de potasio (18 a $99 \mathrm{mg} \%$ ).

Tabla Na 4.- Jugos de frutas $(n=7)$

Potasio y calcio son los minerales que se encuentran en mayor concentración. El jugo de naranja contenía $72 \mathrm{mg} \%$ de potasio y $60 \mathrm{mg} \%$ de calcio. El jugo de toronja, 


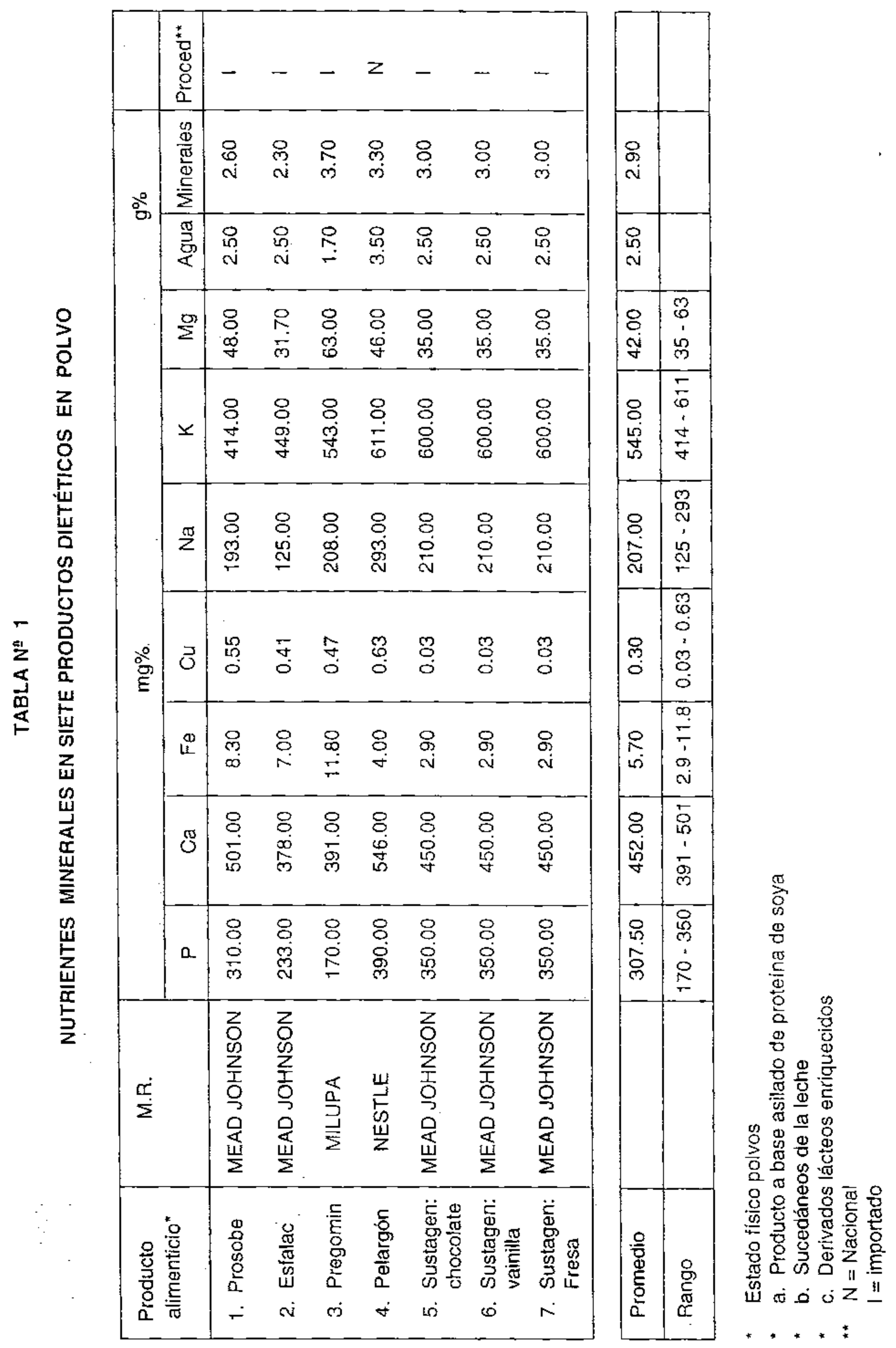




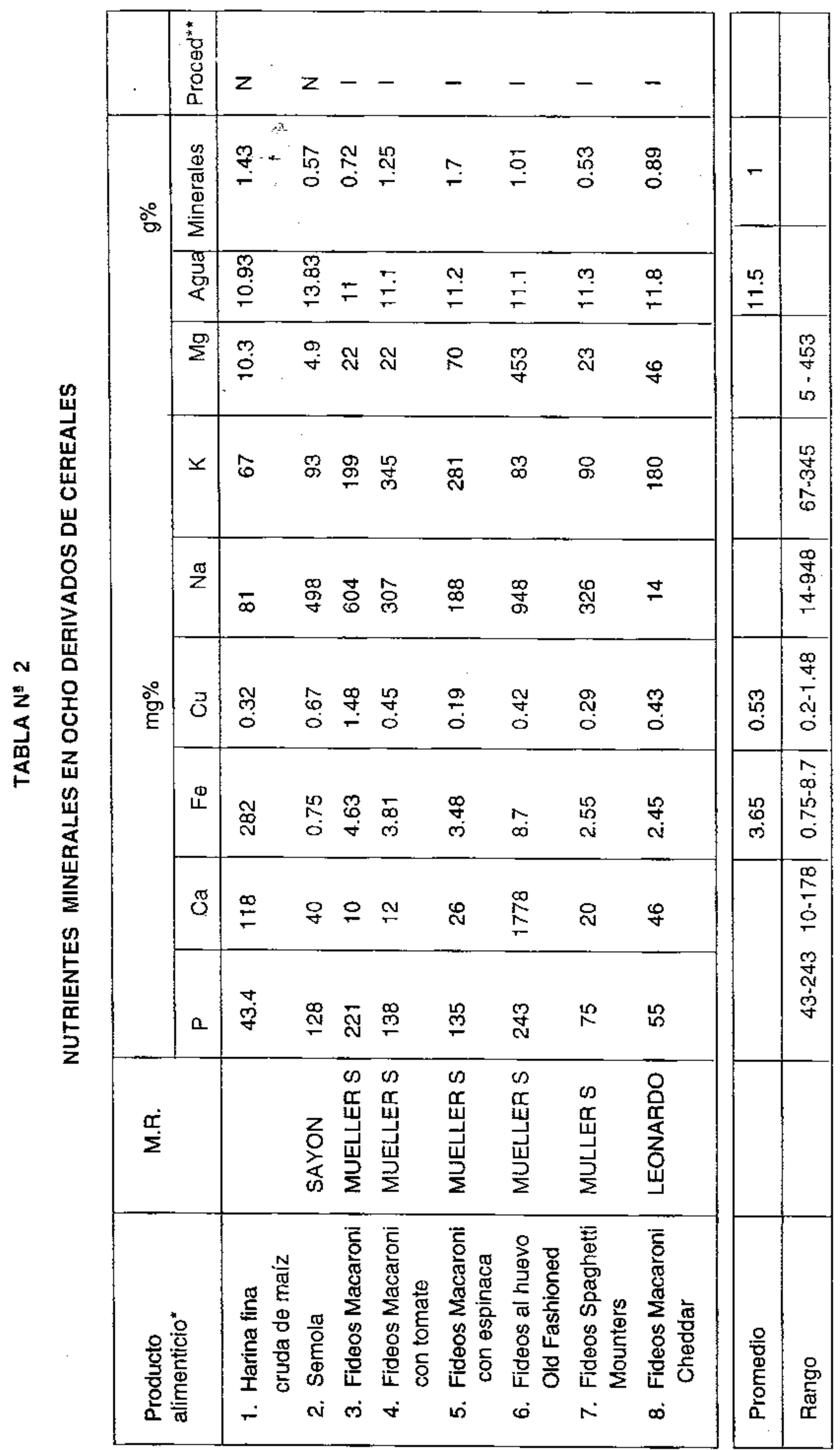

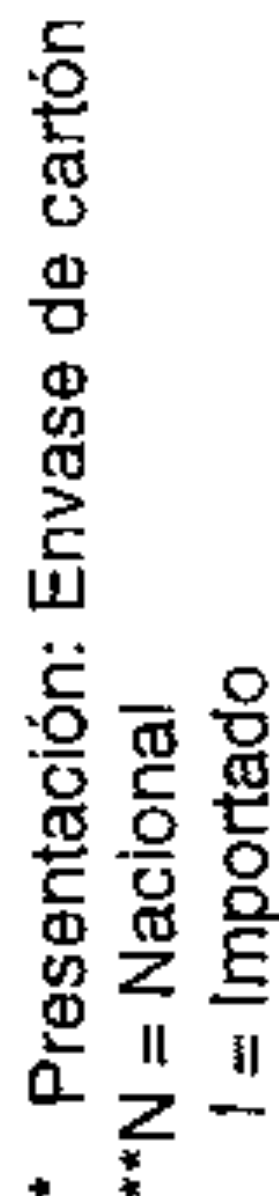




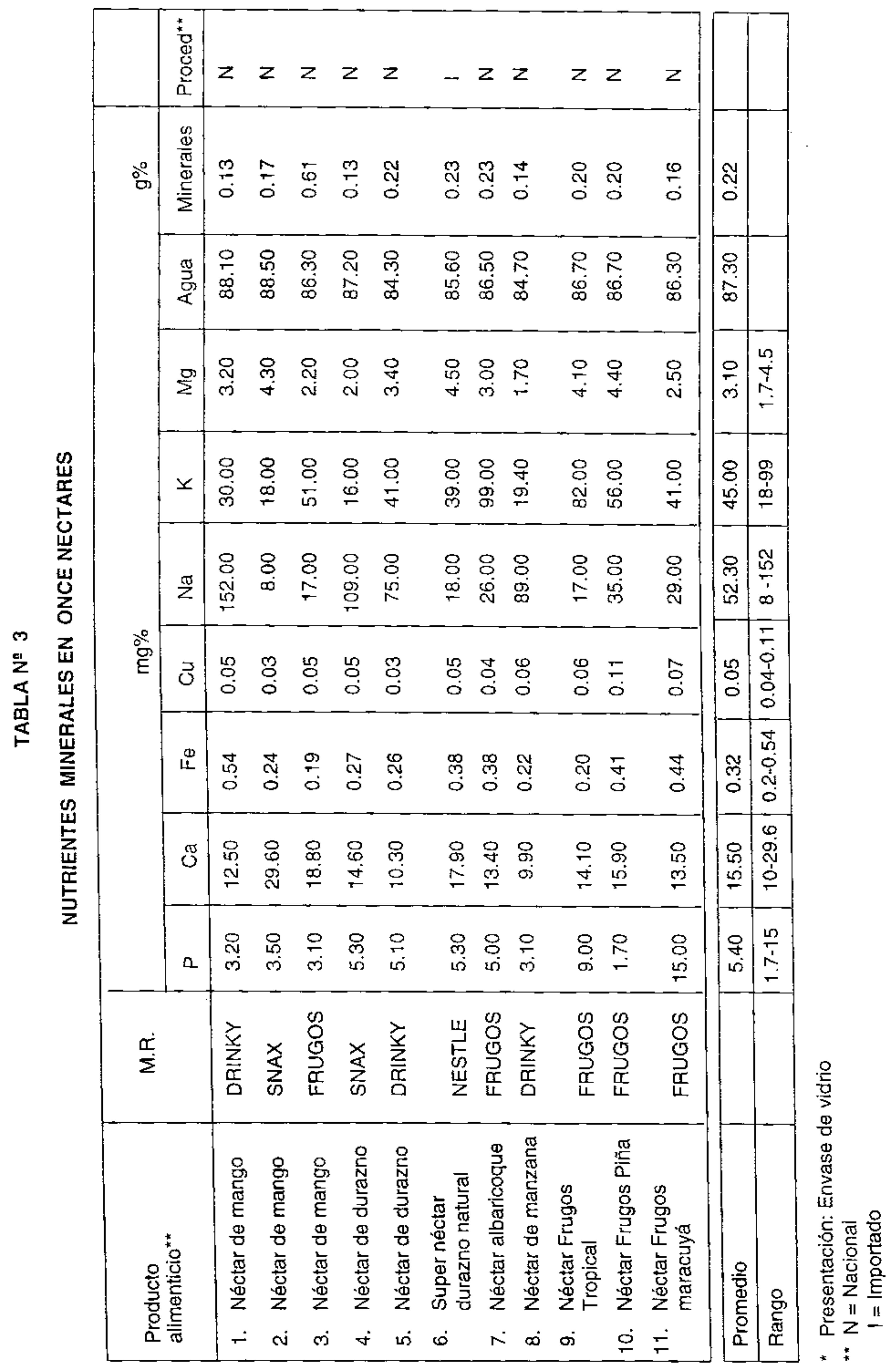




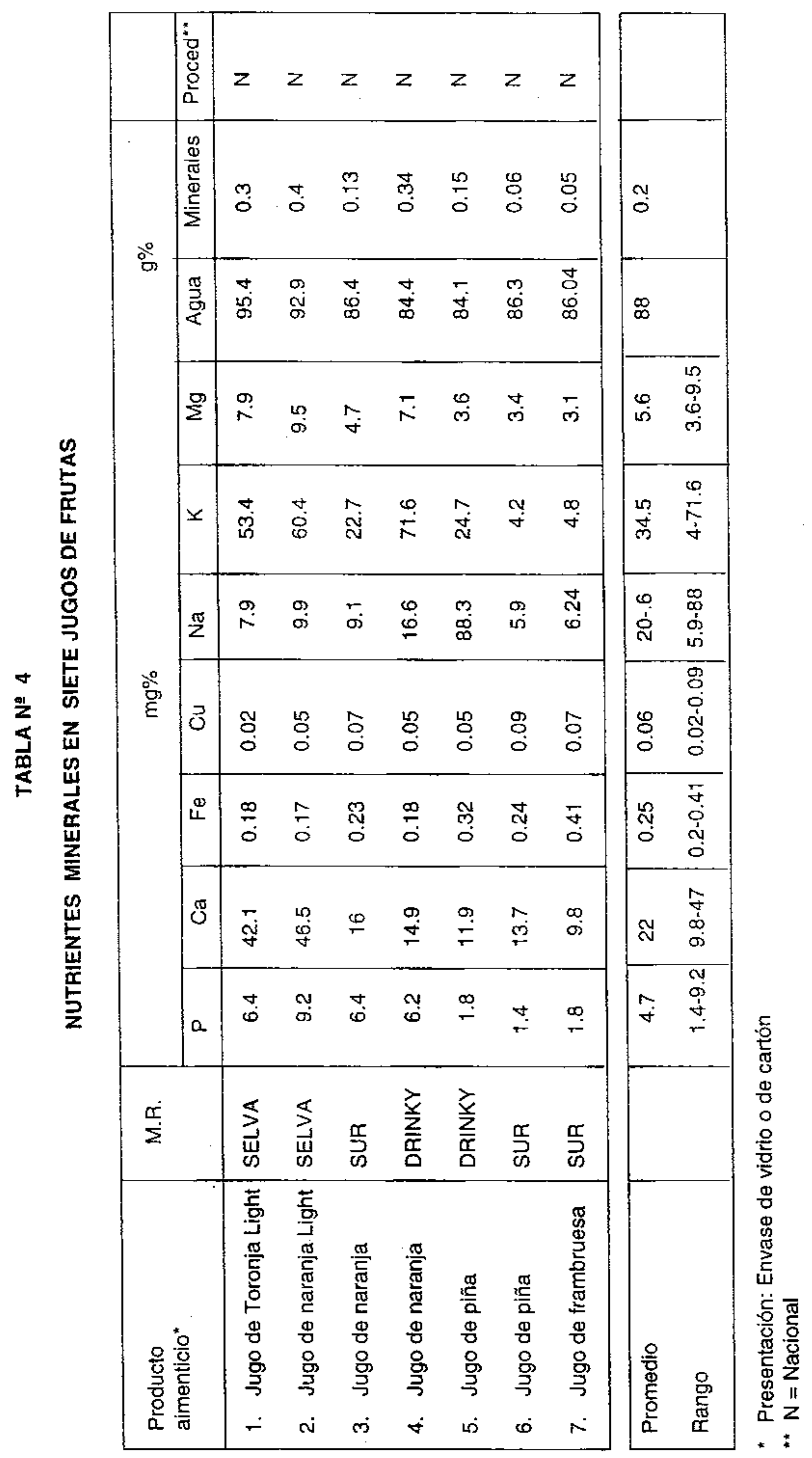


prescntó 53,4 mg\% de potasio y $42 \mathrm{mg} \%$ de magnesio. Cobre y hierro fueron los minerales que se encontraron en menor cantidad: $\mathrm{Cu} 0,02$ a $0,09 \mathrm{mg} \%$, He 0.17 a 0,32 mig.

\section{DISCUSIÓN}

Los nutrientes minerales se encuentran universalmente distribuidos en los alimentos de origen vegetal y animal. Sin embargo, en los alimentos industrializados, que son de consumo habitual en nucstro medio, las concentraciones de estos nutrientes no son conocidas.

Los resultados encontrados en 33 productos indican que éstos contribuyen con cantidades importantes de minerales a las ingestas diarias, las cuales frecuentemente son subestimadas, al no contar con la información.

\section{Calcio y fósforo}

Los contenidos de calcio $(x=452 \mathrm{mg} \%)$ y de fósforo $(x=307.5 \mathrm{mg} \%$ en los productos dictéticos (Tabla $\mathrm{N}^{\circ}$ 1) son concordantes con los valores presentados en leche en polvo. La relación $\mathrm{Ca} / \mathrm{P}$ asegura una buena utilización de calcio y la conservación de su balance.

Entre los derivados de los cereales (Tabla $\mathrm{N}^{\circ} 2$ ), que fueron estudiados, la harina de maíz y los fideos enriquecidos con huevo tenían las mayores concentraciones (118 y $178 \mathrm{~m} \%$ respectivamente). Los trabajos de Paul y Sohgate (4) informan de contenidos de $140 \mathrm{~m} \%$ de calcio en harina de trigo enriquecida.

Los 3 néctares de mango analizados tenían, en promedio, $17 \mathrm{mg} \%$ de calcio; en duraznos, Ios valores se encontraron entre 10 y $17,9 \mathrm{~m} \%(x=14 \mathrm{mg} \%)$; el fósforo -comparativamente- se encuentra en niveles bastante bajos: $1,7 \mathrm{mg} \%$ (néctar de piña $; 3,0 \mathrm{mg} \%$ en mango; $5,0 \mathrm{mg} \%$ en durazno y hasta de $15 \mathrm{mg} \%$ en néctar de maracuyá.

\section{Sodio y potasio}

En la mayoría de los productos alimenticios, la concentración de potasio es mayor que la de sodio. En la presente investigación, el valor promedio de potasio es superior a la concentración de sodio; sólo en el caso de los siete (7) productos dietéticos (545 $\mathrm{mg} \%$ de potasio y $207 \mathrm{mg} \%$ de sodio) y de los siete (7) jugos de frutas $(34,5 \mathrm{mg} \%$ de polasio y $20,6 \mathrm{mg} \%$ de sodio).

\section{Hierro y cobre}

Las Tablas Nacionales de Composición de Alimentos (2) prestan especial atención a los contenidos de hierro en los alimentos.

En este caso, los cuatro grupos de alimentos industrializados estudiados, presentan cantidades relativamente pequeñas de este mineral. Las concentraciones promedio de hierro iban desde $0,25 \mathrm{mg} \%$ (en jugos, Tabla $\mathrm{N}^{\circ} 4$ ), hasta $5,7 \mathrm{mg} \%$ en productos dietéticos (Tabla $\mathrm{N}^{\circ}$ 1); las concentraciones de cobre, desde $0,05 \mathrm{mg} \%$ (néctares, Tabla $\mathrm{N}^{\circ}$ ) hasta $0,53 \mathrm{mg} \%$ en los derivados de cereales (Tabla $\mathrm{N}^{\circ}$ 2).

Teniendo en cuenta que estos minerales son de naturaleza hematógena y que están catalogados dentro de la categoría de nutrientes esenciales, toda contribución a las dietas 
diarias debe ser evaluada, particularmente en el caso de productos que son preferentemente incorporadas a las dietas de los niños.

\section{Magnesio}

A semejanza del sodio y polasio, el magnesio se halla ampliamente distribuido entre los tejidos animales y vegetales; habiéndose informado que los cereales integrales lo contienen en cantidades que superan los $100 \mathrm{mg} \%$. Entre los alimentos que aquí han sido estudiados, en los 7 productos dietéticos, se encontró entre 32 y $63 \mathrm{mg} \%$, con un promedio de $42 \mathrm{mg} \%$ (Tabla $\mathrm{N}^{\circ} 1$ ). En el grupo de derivados de cereales, el promedio fue de $28.3 \mathrm{mg} \%$ (Tabla $\mathrm{N}^{\circ}$ 2), la sémola contiene la menor cantidad (4,9 $\mathrm{mg} \%$ ) y los fideos contienen entre 20 y $70 \mathrm{mg} \%$.

En los procesos biológicos ningún nutriente actúa en forma aislada, su metabolismo es influenciado por el de los otros nutrientes que son ingeridos con al mismo alimento o con el conjunto de alimentos que conforman las dietas. Por ejemplo, cantidades excesivas de fósforo, puede bloquear la hidroxilación de la 25 hidroxi vitamina D3 (3), o interaccionar negativamente con el metabolismo del calcio.

Un exceso de elementos trazas esenciales puede resultar toxico o competir con la absorción de otros elementos esenciales. Por consiguiente, es de vital importancia conocer las cantidades de estos nutrientes en los alimentos para poder establecer su real contribución a las necesidades fisiológicos o para anticipar posibles interacciones que afectarían su utilización biológica.

Adicionalmente, no se descarta la posibilidad de que alimentos industrializados incorporen a su composidión, algún elemento procedente de los equipos empleados en los procesos unitarios.

Todo lo anterior hace necesario investigar la composición de los diferentes alimentos por su contenido de nutrientes minerales, a fin de disponer de datos que permitan determinan los verdaderos aportes nutricionales.

\section{BIBLIOGRAFÍA}

1. Association of Official Agriculture Chemical A.O.A.C. Official Methods of Analysis. Washington D.C. 1980.

2. Collazos Ch., Carlos y colab.-Composición de alimentos de mayor consumo en el Perú. Ministerio de Salud, Instituto Nacional de Nutrición. Lima. Sexta edición 1993.

3. De Luca, H.- Some new concepts from study of the metabolism and function of vitamin D. Nutr. Rev. vol 38: $169-182,1980$.

4. Paul, A. and Southgate, D.A.- McCance and Widdowson's The composition of foods. 4th ed. MRC London and Elsevier, Amsterdam 1978. 